\title{
Fully autocompensating high-dimensional quantum cryptography by optical phase conjugation
}

\author{
Jesús Liñares,, , | Xesús Prieto-Blanco, ${ }^{1}$ Daniel Balado, ${ }^{1}$ and Gabriel M. Carral ${ }^{1}$ \\ ${ }^{1}$ Quantum Materials and Photonics Research Group, Optics Area, \\ Department of Applied Physics, Faculty of Physics / Faculty of Optics and Optometry, \\ University of Santiago de Compostela, Campus Vida $s / n$, \\ E-15782, Santiago de Compostela, Galicia, Spain
}

\begin{abstract}
We present a bidirectional quantum communication system based on optical phase conjugation for achieving fully autocompensating high-dimensional quantum cryptography. We prove that random phase shifts and couplings among $2 N$ spatial and polarization optical modes described by $\mathrm{SU}(2 N)$ transformations due to perturbations are autocompensated after a single round trip between Alice and Bob. Bob can use a source of single photons or, alternatively, coherent states and then Alice attenuates them up to a single photon level, and thus non-perturbated 1-qudit states are generated for high-dimensional QKD protocols, as the BB84 one, of a higher security.
\end{abstract}

Introduction.-Quantum cryptography is based on the properties of quantum mechanics to obtain secure quantum key distribution (QKD) by using different protocols. One of them is the seminal so-called BB84 protocol in which four states define a set of two mutually unbiased basis (MUBs). On the other hand, space division multiplexing has been proposed to further increase the data bandwidth in optical fiber communications [1, and accordingly, high interest has arisen in new optical fibers such as few-mode fibers and multicore fibers. Likewise, optical satellite communications, and in general free space optical communications, based on spatial modes, such as those ones carrying orbital angular momentum, constitutes a promising communications technology [2]. In parallel, the interest in the implementation of quantum cryptography in both these new optical fibers and in free space has also remarkably increased in the last few years. The main reason is that by using spatial optical modes a high-dimensional QKD (HD-QKD) can be implemented, which in turn improves cryptographical security 3. Different optical systems have been proposed to implement QKD cryptography in both optical fibers and free space; such systems can use different kind of modes, for instance, polarization modes in monomode optical fibers [4] and free space [5], collinear spatial modes in few-mode optical fibers [6] and free space [7] and spatial codirectional modes in multicore optical fibers [8]. However, one of the most important drawbacks is that all guided and free space modes need to keep stable over long propagation distances along optical fibers or the atmosphere. Modes undergo instability because light, in its propagation in free space or along optical fibers, finds small spatial perturbations (imperfections) or slow temporal perturbations. This gives rise to random modal coupling (modal crosstalking) which, together with random intermodal phases, causes instability of both modes and quantum states. To overcome this drawback, specific (partial) autocompensating techniques have been

* Corresponding author: suso.linares.beiras@usc.es proposed in bidirectional quantum communication systems. For instance, polarization autocompensating quantum cryptography with 1-qubit states excited in polarization modes 4, 9, or more recently with 1-qudit states excited in spatial modes acquiring random relative phases [10, 11. However, to our knowledge, a fully autocompensating solution has not been proposed.

In this Letter we propose and prove a fully autocompensating quantum cryptography technique based on optical phase conjugation (OPC) and valid for both free space optical communication and optical fiber communications where spatial and polarization modal couplings are not negligible. In a most formal way, by using OPC we compensate for unwanted effects in 1-qudit states caused by an arbitrary number $q$ of unpredictable unitary transformations $\mathrm{SU}(2 N)$, where $2 N$ is the number of spatial modes with two polarizations. For the sake of expositional convenience we present a detailed study for multicore optical fibers (MCF) which can in turn be formally applied to both few-mode optical fibers (FMFs) and free space communications. Input multimode single photon states can be used, or alternatively, coherent states which are attenuated in their way back up to a single photon level (weak coherent states), so that 1-qudit states are produced. In this case, decoy states [12] have also to be generated for security purposes, as usual.

Optical phase conjugator.- Let us consider a four-wave mixing (FWM) non-linear interaction system as shown in Fig. 1 In general, an input spatial multimode optical quantum state $|L\rangle$ propagating along $-z$ direction and with frequency $\omega$ reaches the FWM system; such state emerges from an optical fiber (OF). After the OF a collimating lens (CL) is inserted for collimating the optical fiber modes $\mathbf{e}_{j}(x, y), j=1, \ldots, N$. This CL is not strictly necessary but helps to understand the physical process. After the collimating lens, a polarizing beamsplitter (PBS) separates horizontal and vertical polarization modes ( $x$ and $y$-modes). The linear $x$-polarization mode is rotated $\pi / 2$ by means of a $\mathrm{HWP}_{\pi / 4}$ (HWP rotated $\pi / 4)$. Finally, we have a third order non linear material of length $l$ in which it is considered that there are 


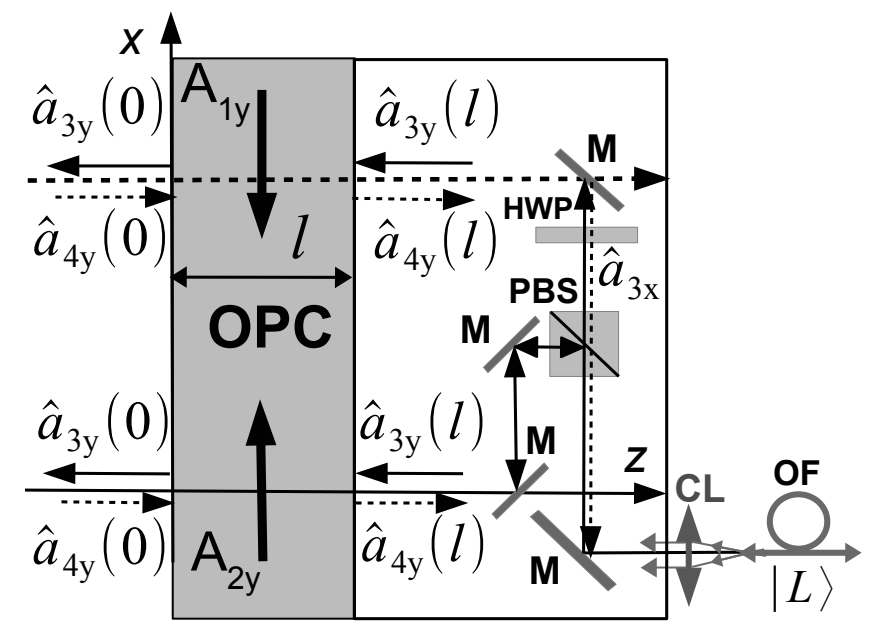

FIG. 1. Four-wave mixing system with two pump waves $\mathrm{A}_{1 y}$ and $\mathrm{A}_{2 y}$ and an input state $|L\rangle$ emerging from an optical fiber $(\mathrm{OF})$ along $-z$. Optical fiber modes are collimated by a lens (CL) and redirected by mirrors (M). PBS separates polarizations, and vertical or $x$-polarization becomes vertical or $y$-polarization by using a HWP rotated $\pi / 4$. Finally, the nonlinear medium implements an optical phase conjugation of the reflected modes $\hat{a}_{4 y}$.

two strong vertically polarized counter-propagating intense pump modes (strong coherent states) of frequency $\omega$ and amplitudes $\mathrm{A}_{1 y}$ and $\mathrm{A}_{2 y}$. A possible isotropic non linear material for implementing $\mathrm{OPC}$ can be $\mathrm{CS}_{2}$ [14, which would contribute for vertical polarization with the term $\chi_{y y y y}^{(3)}$ of the third order non linear tensor. For the moment, let us consider that $|L\rangle$ is a single mode state excited in the incident mode 3 coming from an OF and with an associated optical field operator $\hat{\mathrm{E}}_{3 y} \propto \hat{a}_{3 y}$, with $\hat{a}_{3 y}$ an absorption operator. When $|L\rangle$ reaches the non linear material then a fourth mode (reflected mode 4) arises, with an associated field operator $\hat{\mathrm{E}}_{4 y} \propto \hat{a}_{4 y}$. We are interested on the quantum states propagating along $z$-direction in modes 3 and 4 (idler and signal modes) after non linear interaction, that is, in spatial propagation not in temporal evolution (Hamiltonian operator) [13. Therefore, for spatial nonlinear coupling is convenient to use the Momentum operator describing the quantum mode interaction [13 15],

$$
\hat{M}_{I}=\int \chi_{y y y y}^{(3)} \mathrm{E}_{1 y} \mathrm{E}_{2 y} \hat{\mathrm{E}}_{3 y} \hat{\mathrm{E}}_{4 y} d x d y d t
$$

where the intense pump waves 1 and 2 can be treated clasically and modes 3 and 4 in a quantum way, that is,

$$
\begin{array}{r}
\mathrm{E}_{\left(\begin{array}{c}
1 y \\
2 y
\end{array}\right)}=\mathrm{A}_{\left(\begin{array}{c}
1 y \\
2 y
\end{array}\right)} e^{\mp i k_{o} n x} e^{-i \omega t}+c . c ., \\
\hat{\mathrm{E}}_{\left(\begin{array}{c}
3 y \\
4 y
\end{array}\right)}=\sqrt{\hbar \omega} \mathrm{e}_{j}^{\left(\begin{array}{c}
3 \\
4
\end{array}\right)}(x, y) \hat{a}_{\left(\begin{array}{c}
3 y \\
4 y
\end{array}\right)} e^{\mp i k_{o} n z} e^{-i \omega t}+\text { h.c., }
\end{array}
$$

where $\mathrm{e}_{j}$ is a $j$ th normalized spatial mode of the OF with $x$ or $y$-polarization. As these modes are collimated by the CL then $\mathrm{e}_{j}^{(3)} \approx \mathrm{e}_{j}^{(4)}$. By inserting the above pump waves and operators into Eq. (1) and performing the temporal integrations the following operator is found

$$
\hat{M}_{I}=\hbar \chi_{e f f} \mathrm{~A}_{1 y} \mathrm{~A}_{2 y} \hat{a}_{3 y}^{\dagger} \hat{a}_{4 y}^{\dagger}+\text { h.c. },
$$

where $\chi_{\text {eff }}$ is an effective non-linear susceptibility which groups together all physical constants. From this operator the spatial Heisenberg equations [13] can be obtained,

$$
-i \hbar \frac{\partial \hat{a}_{m y}}{\partial z}=\left[\hat{a}_{m y}, \hat{M}_{I}\right]
$$

where $m=3,4$. Let us denote the input operators as $\hat{a}_{3 y}(l) \equiv \hat{a}_{o 3}$ and $\hat{a}_{4 y}(0) \equiv \hat{a}_{o 4}$, and the output operators as $\hat{a}_{3 y}(0) \equiv \hat{a}_{3}$, associated to the optical mode transmitted along system, and $\hat{a}_{4 y}(l) \equiv \hat{a}_{4}$, associated to the reflected optical mode (see Fig. 1). As mentioned, there are two pump waves with very large amplitudes $\mathrm{A}_{1 y}$ and $\mathrm{A}_{2 y}$ and initial phases equal to zero, then the non-linear interaction strength is given by a coupling coefficient $\kappa=\chi_{\text {eff }}\left|\mathrm{A}_{1 \mathrm{y}}\right|\left|\mathrm{A}_{2 \mathrm{y}}\right|$, therefore the coupling is parametrically governed by $\left|A_{1 y}\right|\left|A_{2 y}\right|$, that is, the efficiency of the process is governed by pumping. It is easy to check that the solutions of the spatial Heisenberg equations obtained by inserting Eq. (4) into Eq. (5) provide the well-known operator transformations [14, 16]

$$
\begin{gathered}
\hat{a}_{3 y}(l) \equiv \hat{a}_{o 3}=\sec (\kappa l) \hat{a}_{3}+i \tan (\kappa l) \hat{a}_{4}^{\dagger} \\
\hat{a}_{4 y}(0) \equiv \hat{a}_{o 4}=\sec (\kappa l) \hat{a}_{4}+i \tan (\kappa l) \hat{a}_{3}^{\dagger} .
\end{gathered}
$$

Next, let us consider a coherent state $|L\rangle=\left|\alpha_{3} 0_{4}\right\rangle$ excited in an optical fiber mode. By taking into account the complex displacement operator and using Eq. (6) the output state can be rewritten as follows

$$
|L\rangle=e^{\alpha \hat{a}_{o 3}^{\dagger}-\alpha^{\star} \hat{a}_{o 3}}|00\rangle \rightarrow\left|L_{c}\right\rangle=|s \alpha\rangle\left|-i t \alpha^{\star}\right\rangle,
$$

where $s=\sec (\kappa l)$ and $t=\tan (\kappa l)$. Note that the reflected coherent state has been conjugated, that is, the FWM is an OPC; besides, quantum-mechanically the OPC means that $\hat{a}_{o 3} \propto \hat{a}_{4}^{\dagger}$. Let us recall, however, that we are interested in multimode coherent states, that is, $|L\rangle=\left|\alpha_{1} \ldots \alpha_{N}\right\rangle$ excited in $N$ optical modes, therefore, the OPC produces the multimode coherent state $\left|L_{c}\right\rangle=$ $\left(\left|s \alpha_{1}\right\rangle\left|-i t \alpha_{1}^{\star}\right\rangle\right) \ldots\left(\left|s \alpha_{N}\right\rangle\left|-i t \alpha_{N}^{\star}\right\rangle\right)$. Alternatively, we could have a single photon source, therefore we can generate an input 1 -qudit state $\left|L_{o}\right\rangle=\sum_{o 3 j} c_{j}\left|1_{o 3 j}\right\rangle$, with, $j=1, \ldots N$, where the subindex $o 3 j$ indicates that the $N$ modes are incident on the OPC. By taking into account that $\left|1_{o 3 j}\right\rangle=\left(\hat{a}_{o 3 j}^{\dagger}+\hat{a}_{o 3 j}\right)|0\rangle$, we obtain the output quantum state $|L\rangle=s \sum_{3 j} c_{j}\left|1_{3 j}\right\rangle+i t \sum_{4 j} c_{j}\left|1_{4 j}\right\rangle$.

Autocompensation with codirectional modes.-First of all and for the sake of expositional convenience, we provisionally assume that polarization is maintained under propagation (we will consider spatial and polarization couplings later, although in atmosphere and special optical fibers polarization can be maintained), therefore we only consider coupling among $N$ spatial modes 
with the same linear polarization. In particular, let us consider codirectional modes of a MCF with $N$ modes (cores) whose propagation constants are $\beta_{o i}, i=1, \ldots, N$ and with associated absorption operators $\hat{a}_{i}$. We must stress that the results that we are going to obtain are also valid for collinear modes of FMF or a free space optical modes. The quantum state reaching Bob system from the Alice system will be an unpredictable quantum state, and as a consequence, modal coupling prevents us to implement any QKD protocol. Next, we show how to overcome this drawback by OPC. Let us consider a perturbation $P_{s}(x, y)$ that induces modal coupling and can be considered as $z$-invariant along a distance $s$, then the spatial Heisenberg equation describing modal coupling among $N$ optical field operator modes $\hat{E}_{i} \propto \hat{a}_{i}$ can be written as follows 15.

$$
-i \hbar \frac{\partial \hat{a}_{i}}{\partial z}=\hbar\left\{\beta_{i} \sum_{j=1}^{N} \delta_{i j} \hat{a}_{j}+\sum_{j \neq i}^{N} \kappa_{i j} \hat{a}_{j}\right\} \equiv \hbar \sum_{j=1}^{N} C_{i j} \hat{a}_{j},
$$

here $\beta_{i}=\beta_{o i}+\kappa_{i i}$ are the perturbed propagation constants due to (random) modal selfcoupling $\left(\kappa_{i i}\right)$, and $\kappa_{i j}$ are (random) modal coupling coefficients due to cross coupling. From a most fundamental point of view, an arbitrary coupling coefficient of spatial modes $i, j$ is given by $\kappa_{i j}=\int \mathrm{e}_{i}(x, y) P_{s}(x, y) \mathrm{e}_{j}(x, y) d x d y$, where $\mathrm{e}_{(i, j)}(x, y)$ are the mode amplitudes. Note that $\kappa_{i j}=\kappa_{j i}$ and therefore $\left[C_{i j}\right] \equiv C$ is a symmetric matrix. Therefore, by using the algebraic properties of symmetric matrices, the formal matrix solution $\left[S_{i j}\right] \equiv S=\exp \{i C z\}$ of differential equation in Eq. (9) is a complex symmetric matrix, that is, $S_{i j}=S_{j i}$. On the other hand, modal coupling is an unitary transformation, therefore $\left[S_{i j}\right]^{-1}=\left[S_{j i}\right]^{\star}=\left[S_{i j}\right]^{\star}$. In general, we will have an arbitrary number $q$ of perturbations, accordingly, the total effect along $z$ direction from a system $\mathrm{B}$ (Bob) to system A (Alice) can be represented by the total matrix $M=S_{1} \cdots S_{q}$. Then, if we have an OPC at A, the matrix $M$ becomes $M^{\star}=S_{1}^{\star} \cdots S_{q}^{\star}$. Now, the quantum state is propagated back to system $\mathrm{B}$, therefore we have to use a reflected coordinate system which is defined, without loss of generality, by $(-x) y(-z)$ with respect to the incident coordinate system $x y z$. The coupling coefficients $\kappa_{i j}$ are invariant under the transformation $x \rightarrow-x$ and consequently the matrices $C$ are also invariant. Once the light has travelled the path back and forth, the total coupling matrix is $M^{t} M^{\star}=\mathbb{I}$, with super index $t$ indicating transpose. Then, the unpredictable modal coupling has been removed. In short, if Bob launches a state $|L\rangle$ undergoing modal coupling along an $\mathrm{OF}$ or in the atmosphere, the state after the OPC and traveling its way back is $\left|L_{c}\right\rangle=\left(\left|s \alpha_{1}\right\rangle\left|-i t \alpha_{1}^{\star}\right\rangle\right) \ldots\left(\left|s \alpha_{N}\right\rangle\left|-i t \alpha_{N}^{\star}\right\rangle\right)$, that is, we recover the initial state except phases $\pi$ and conjugations.

Autocompensation with spatial and polarization modes.-As commented, we also have to remove unpredictable polarization modal coupling together with the above spatial modal coupling. First of all we characterize the matrix transformation produced by an arbitrary coupling between linearly polarized spatial modes (e.g., LP modes). Since there are $2 N$ modes we consider the new subindices $i, j=\{1 H, 1 V, 2 H, 2 V, \ldots, N H, N V\}$, with $H \equiv x, V \equiv y$. As in the above (scalar) case, the coupling matrix $\left[C_{i j}\right] \equiv C$ is also a complex symmetric matrix. However, when considering back propagation, this matrix gets modified because, as commented, the incident coordinate system $x y z$ becomes $(-x) y(-z)$ under reflection. Indeed, an arbitrary coupling coefficient of spatial modes $m, n$ with different polarization is given by $\kappa_{m H n V}=\int \mathrm{e}_{m H}(x, y) P_{v}(x, y) \mathrm{e}_{n V}(x, y) d x d y$, where $P_{v}(x, y)$ is an arbitrary perturbation producing polarization (vector) modal coupling. Obviously, under reflection (back path) we have $\mathrm{e}_{n H}(x, y) \rightarrow-\mathrm{e}_{n H}(x, y)$, then $\kappa_{i j} \equiv \kappa_{m H n V} \rightarrow-\kappa_{m H n V} \equiv-\kappa_{i j}$. Note that for the same polarization the coupling coefficient is positive (or zero) under reflection. Therefore, the coupling matrix $\left[B_{i j}\right] \equiv B$ under reflection can be written as follows

$$
B=\left(I_{N} \otimes \sigma_{z}\right) C\left(I_{N} \otimes \sigma_{z}\right) \equiv D C D,
$$

with $I_{N}$ the $N$-dimensional identity matrix, $\otimes$ the tensor product and $\sigma_{z}$ the third Pauli matrix. Next, by taking into account that $D D=\left(I_{N} \otimes \sigma_{z}\right)\left(I_{N} \otimes \sigma_{z}\right)=I_{2 N}$ $\left(I_{2 N}\right.$ the identity matrix $\left.2 N \times 2 N\right)$, it is easy to check that the transformation matrix produced by the perturbation $P_{v}(x, y)$, that is, the formal matrix solution $\left[R_{i j}\right] \equiv R=\exp \{i B z\}$ can be written, by using the Taylor expansion of an exponential function, as follows

$$
R=\left(I_{N} \otimes \sigma_{z}\right) S\left(I_{N} \otimes \sigma_{z}\right) \equiv D S D .
$$

where $S=\exp \{i C z\}$. Note that matrix $R$ (transformation of the absorption operators $\hat{a}_{j H}, \hat{a}_{j V}$ ) is also symmetric. On the other hand, it is also easy to check that the $\mathrm{HWP}_{\pi / 4}$ introduces a phase $\pi$ between $H$-mode and $V$-mode of every spatial mode in its way back. Therefore, after OPC system, when polarization modes are recombined in the PBS (see Fig. 1), the matrix $D=I_{N} \otimes \sigma_{z}$ is implemented. Consequently, by considering the general case of $q$ random couplings, we obtain, after the path back to Bob, the total matrix

$$
M_{T}=R_{q} \cdots R_{1} D S_{1}^{\star} \cdots S_{q}^{\star}=D,
$$

where we have taken into account the following relationships $R_{k}=D S_{k} D, D D=I_{2 N}$ and $S_{k} S_{k}^{\star}=I_{2 N}, k=1, \ldots q$. In short, symmetric spatial perturbations together with polarization perturbations have been removed.

General SU(2N) autocompensation.-The above results have made clear autocompensation of symmetric unitary coupling transformations. Now, we generalize the above results for non symmetric unitary coupling transformations, for example, rotations due to optical activity $(N=1), 2 N \times 2 N$ abstract rotations and so on, that is, $\mathrm{SU}(2 N)$ perturbations. In order to prove this assertion we must take into account that all unitary transformation $\mathrm{SU}(2 \mathrm{~N})$ can be factorized as a ordered product of $\mathrm{SU}(2)$ transformations of subspaces $i, j$ [17, 18. Thus, 
by proving autocompensation by OPC of an arbitrary $\mathrm{SU}(2)$ transformation the case $\mathrm{SU}(2 N)$ is also proven. A general $\mathrm{SU}(2)$ transformation $S$ can be represented by

$$
S=\left(\begin{array}{cc}
\cos \theta & i \sin \theta e^{-i \delta} \\
i \sin \theta e^{i \delta} & \cos \theta
\end{array}\right) \equiv Z(\delta) X(\theta) Z(-\delta)
$$

with $Z( \pm \delta)=\operatorname{diag}\left(1, e^{ \pm i \delta}\right)$ the matrix of a phase retarder $\pm \delta$ generated by the the Pauli's matrix $\sigma_{z}$, and $X(\theta)$ a matrix whose generator is the Pauli's matrix $\sigma_{x}$, with elements $X_{11}(\theta)=X_{22}(\theta)=\cos \theta$, and $X_{12}(\theta)=X_{21}(\theta)=i \sin \theta$. By considering that we are in a polarization two-mode subspace, it is easy to check that the matrix by reflection is characterized by the changes $\delta, \theta \rightarrow-\delta,-\theta$, then $T=D_{2} S^{T} D_{2}$, where $D_{2}=\sigma_{z}$. Therefore, after OPC and by taking into account the action of the $\mathrm{HWP}_{\pi / 4}\left(D_{2}\right)$ we obtain the following result

$$
T S^{\star}=D_{2} S^{T} D_{2} D_{2} S^{\star}=D_{2} S^{T} S^{\star}=D_{2},
$$

and then autocompensation is again achieved. Rigorously, a general SU(2) matrix requires to consider an additional matrix corresponding to a $\alpha$-phase retarder, that is, $Z(\alpha) S$; however, a retarder is also autocompensated, as proven above. We must also stress that similar results are found for spatial two-mode subspace. Likewise, topological phases due to helical paths, torsions and so on of an OF can be also autocompensated because such phases also correspond to unitary transformations (rotations and so on) [21]. In short, a multimode coherent state $|L\rangle=\left|\alpha_{1 H} \alpha_{1 V} \ldots \alpha_{N H} \alpha_{N V}\right\rangle$ coming from Bob, becomes a predictable reflected multimode coherent state $\left|L_{c}\right\rangle=\left|-i t \alpha_{1 H} i t \alpha_{1 V} \ldots-i t \alpha_{N H} i t \alpha_{N V}\right\rangle$, therefore, we have proved that OPC has canceled a number $q$ of unpredictable perturbations represented by $\mathrm{SU}(2 N)$ transformations.

Optical fiber setup for HD-QKD.-By taking into account the results obtained we can implement an autocompensating optical system for HD-QKD BB84 quantum cryptography in optical fibers. Such a system is shown in the sketch of Fig. 2. Its optical configuration is as follows: the first device is a coherent states generator (CSG) located in Bob system, which emits coherent states $|L\rangle=\left|\alpha_{1 H} \alpha_{1 V} \ldots \alpha_{N H} \alpha_{N V}\right\rangle$ to be excited in $2 N$ optical modes of a MCF (or a FMF fiber, or free space), with $\alpha_{1 H}=\ldots=\alpha_{N V}$, for generating quantum states belonging to MUBs. Next, optical fiber delayers $\left(\mathrm{OFD}_{1}\right)$ produce modal delays $\tau_{j}(j=1, \ldots, 2 N)$, that is, we have a multimode coherent state formed by the tensor product of delayed single mode coherent states. These delays will allow to Alice introduce phases in each spatial mode. Afterwards, a set of optical circulators SOC launches the state towards Alice system. Besides, a multiplexing/demultiplexing (MUX/DEMUX) device is also needed if collinear propagation is required as in a FMF or even in free space. Different spatial multiplexing devices can be implemented according to the kind of collinear modes [19, 20]. Note that If we use a MCF then the MUX/DEMUX device is not required (codirectional modes). After propagation along the OF each $j$ th

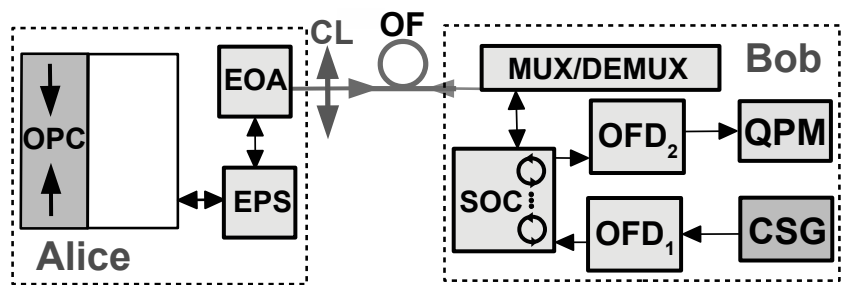

FIG. 2. Basic optical fiber setup for autocompensating HDQKD by OPC (see text under Optical fiber setup for $H D-Q K D$ for description).

delayed single mode coherent state (excited in each core of a MCF) becomes a multimode coherent state due to modal coupling. Such an state reaches the OPC device described in detail in Fig. 1 and explained above. Note that now we have placed an electro-optic phase shifter (EPS) and an electro-optic attenuator (EOA) [10] in offposition between the OPC and the CL-OF (see Fig. 2). We must stress that after the OPC the reflected state has to be coupled again to the OF, however, the OPC implements by itself this transverse modal coupling to the OF (analogous to the well-known image restoration by OPC [14, 16]), although different systems can be used to optimize this coupling, for example, as in our case, by means of a CL. Next, the reflected state in the OPC device goes through the EPS which introduces global phases $\theta_{j}$ on the mentioned multimode coherent states, and the EOA attenuates the state up to single photon level (note that at Bob system each of these multimodes states will become again a $j$ th delayed single mode coherent state due to OPC). The purpose of the EOA is not only to attenuate but also to increase the security of the system, i.e., the attenuation of the EOA can be controlled, enabling the production of different attenuated pulses: signal and decoy states against different attacks of an eavesdropper Eve, as in the photon-number-splitting attack, although we must stress that modal coupling is also a defense against attacks in line. Therefore, Alice system generates 1-qudit states which propagate along the $\mathrm{OF}$ up to the Bob system and thus the modal coupling and relative phases are fully removed. Next, the set of optical circulators SOC sends the 1-qudit state to the $\mathrm{OFD}_{2}$ device which cancels the delays $\tau_{j}$ between states $\left|1_{j}\right\rangle$ of the 1-qudit. In short, the quantum state generated is

$$
\left|L_{c}\right\rangle \approx \frac{-i}{\sqrt{2 N}}\left\{\sum_{j=1}^{N} e^{i \theta_{j H}}\left|1_{j H}\right\rangle-\sum_{j=1}^{N} e^{i \theta_{j V}}\left|1_{j V}\right\rangle\right\} .
$$

These states allow to implement pairs of $2 N$-dimensional MUBs 3 for QKD. Finally, the state reaches a quantum projective measurer (QPM). Since both QMP and CSG are relevant devices it is worth showing a possible physical implementation of them. A CSG can be easily made by integrated devices with concatenated directional couplers $2 \times 2$, that is, each output of a coupler is connected to other coupler and so on [3, 10. Such couplers can be represented by matrices $X(\theta)$ such as the one presented in 
Eq. (13) where $\theta=\kappa d$ with $\kappa$ a linear coupling coefficient and $d$ the coupling distance. We start from a singlemode coherent state $\left|L_{s m}\right\rangle=|\beta\rangle$, and by modal coupling with concatenated directional couplers $X(\pi / 4)$ a multimode state $\left|L_{s m}^{\prime}\right\rangle=|\alpha\rangle|i \alpha\rangle|-\alpha\rangle|i \alpha\rangle \ldots|-\alpha\rangle|-i \alpha\rangle|-\alpha\rangle|i \alpha\rangle$ is obtained, where $\alpha=2^{-N / 2} \beta$. The relative phases $\{ \pm \pi / 2, \pi\}$ can easily be cancelled by using the EPS of the Alice system when the proper phases $\theta_{1 H}, \ldots \theta_{N V}$ are introduced, then $|L\rangle=|\alpha\rangle \ldots|\alpha\rangle$. As to the QPM, a passive integrated quantum projective measurer that randomly selects bases of dimension $N=2^{m}$ in MCFs has been recenlty proposed by using $X(\pi / 4)$ and $X(\pi / 2)$ couplers and phase shifters $Z(\delta)$ [10]. Obviously, if we had used single photon sources both the attenuation and decoy states would not be required. Finally, we must indicate that bidirectional QKD systems are subject to new lateral attacks like the phase-remapping (PR) one. Likewise, recent security analysis considering such an attack have been made [10, 11, and it was shown that PR can not reduce the QBER bellow the minimum in which a secret key rate is guaranteed under a cloning attack in a normal one-way system. Therefore, bidirectionality does not necessarily make the system more vulnerable.

Free space optical communications.-As commented, free space optical communications can be considered as a problem of $2 N$ collinear modes. As in the case of FMFs, the generation and measurement of quantum states have to be made by a MUX/DEMUX process. A detailed study of this case would incorporate mode diffraction, however in most of cases it can be reduced thanks to the high directionality of lasers, or it can simply be ignored since OPC also compensates diffraction (note that a diverging wave incident on an OPC becomes a converging wave).

Summary.- We have presented a fully autocompensating technique based on optical phase conjugation for high-dimensional quantum cryptography in optical fibers and free space. A single round trip allows to autocompensate the undesired modal coupling and random phase shifts among spatial and polarization modes, and thus HD-QKD protocols such as the BB84 can be implemented.

Authors wish to acknowledge the financial support of this work by Xunta de Galicia, Consellera de Educacin, Universidades e FP, with a grant ConsolidationGRC Ref.-ED431C2018/11, grant Strategic Grouping of Materials (AeMAT) Ref.-ED431E 2018/08, and a predoctoral grant (D. Balado, 2017), co-financied with the European Social Fund.
[1] N. Bai, E. Ip, Y. Huang, E. Mateo, F. Yaman, M. Li, S. Bickham, S. Ten, J. Liñares, C. Montero, V. Moreno, X. Prieto, V. Tse, K. M. Chung, A. P. T. Lau, H. Tam, C. Lu, Y. Luo, G. Peng, G. Li, and T. Wang, Modedivision multiplexed transmission with inline few-mode fiber amplifier, Opt. Express 20, 2668 (2012)

[2] L. Zou, X. Gu and L. Wang, High-dimensional free-space optical communications based on orbital angular momentum coding, Opt. Commun. 410333 (2018)

[3] Y. Ding, D. Bacco, K. Dalgaard, X. Cai, X. Zhou, K. Rottwitt, and L. K. Oxenlw, High-dimensional quantum key distribution based on multicore fiber using silicon photonic integrated circuits, npj Quantum Inf. 3, 25 (2017)

[4] A. Muller, T. Herzog, B. Huttner, W. Tittel, H. Zbinden, and N. Gisin, Plug and play systems for quantum cryptography, Appl. Phys. Lett. 70, 793 (1997)

[5] R. Bedington, J.M. Arrazole and A. Ling, Progress in satellite quantum key distribution, npj Quantum Information 330 (2017)

[6] D. Cozzolino, D. Bacco, B. Da Lio, K. Ingerslev, Y. Ding, K. Dalgaard, P. Kristensen, M. Galili, K. Rottwitt, S. Ramachandran, and L. K. Oxenlwe, Orbital angular momentum states enabling fiber-based high-dimensional quantum communication, Phys. Rev. Appl. 11, 064058 (2019)

[7] D.Jin, Y. Guo, Y. Wang amd D. Huang, Parameter estimation of orbital angular momentum based continuousvariable quantum key distribution, J. Appl.Phys. 127, $213102(2020)$

[8] G. Cañas, N. Vera, J. Carie, P. Gonzlez, J. Cardenas, P. W. R. Connolly, A. Przysiezna, E. S. Gmez, M. Figueroa, G. Vallone, P. Villoresi, T. Ferreira da Silva, G. B. Xavier, and G. Lima, High- dimensional decoy-state quantum key distribution over multicore telecommunication fibers, Phys. Rev. A 96, 22317 (2017)

[9] D. S. Bethune, W. P. Risk, Autocompensating Quantum Cryptography, New J. Phys. 4, 42 (2002)

[10] D. Balado, J. Liñares, X. Prieto-Blanco, D.Barral, Phase and polarization autocompensating N-dimensional quantum cryptography in multicore optical fibers, JOSA B 36, 2793 (2019)

[11] D. Balado, J. Liñares, and X. Prieto-Blanco, Phase autocompensating high-dimensional quantum cryptography in elliptical-core few-mode fibers, J. Mod. Opt. 66, 947 (2019)

[12] W. Hwang, Quantum Key Distribution with High Loss: Toward Global Secure Communication, Phys. Rev. Lett. 91, 057901 (2003)

[13] Y. Ben-Aryeh and S. Serulnik, The quantum treatment of propagation in non-linear optical media by the use of temporal modes, Phys Lett A 155, 473 (1991)

[14] D. M. Pepper, D. Fekete and A. Yariv, Observation of amplified phase-conjugate reflection and optical parametric oscillation by degenerate four wave mixing in a transparent medium, Appl. Phys. Lett. 33, 41 (1978)

[15] J. Liñares, M. C. Nistal and D. Barral, Quantization of Coupled 1d Vector Modes In Integrated Photonics Waveguides, New J. Phys. 10, 063023 (2008)

[16] L. Mandel and E. Wolf, Optical Coherence and Quantum Optics (Cambridge University Press, Cambridge, 1995)

[17] M.Reck, A Zeilinger, H. Bernstein and P. Bertani, Experimental realization of any discrete unitary operator, Phys. Rev. Lett. 73, 58 (1994)

[18] H. de Guise, O. Di Matteo and L. L. Sánchez-Soto, Simple factorization of unitary transformations, Phys. Rev. A 97, 022328 (2018) 
[19] J. Liñares, X. Prieto-Blanco, C. Montero-Orille and V. Moreno, Spatial mode multiplexing/demultiplexing by Gouy phase interferometry, Opt. Lett. 42, 93 (2017)

[20] J. Leach, M.J. Padgett, S. M. Barnett, S. Franke-Arnold, and J.Courtial, Measuring the orbital angular momentum of a single photon, Phys. Rev. Lett. 88, 257901 (2002)

[21] M. V. Berry, Interpreting the anholonomy of coiled light, Nature 326, 266 (1987) 